\title{
Phylogenetic grouping, epidemiological typing, analysis of virulence genes, and antimicrobial susceptibility of Escherichia coli isolated from healthy broilers in Japan
}

\author{
Mototaka Hiki ${ }^{1 *}$, Masaru Usui ${ }^{2}$, Tetsuo Akiyama ${ }^{1}$, Michiko Kawanishi ${ }^{1}$, Mai Tsuyuki ${ }^{1,3}$, Saiki Imamura ${ }^{1,3}$, \\ Hideto Sekiguchi ${ }^{3}$, Akemi Kojima ${ }^{1}$ and Tetsuo Asai ${ }^{4}$
}

\begin{abstract}
Background: The aim of our study was to investigate the possible etiology of avian colibacillosis by examining Escherichia coli isolates from fecal samples of healthy broilers.

Findings: Seventy-eight E. coli isolates from fecal samples of healthy broilers in Japan were subjected to analysis of phylogenetic background, virulence-associated gene profiling, multi-locus sequence typing (MLST), and antimicrobial resistance profiling. Phylogenetic analysis demonstrated that 35 of the 78 isolates belonged to group A, 28 to group B1, one to group B2, and 14 to group D. Virulence-associated genes iutA, iss, $C V a C$, tsh, iroN, ompT, and hlyF were found in 23 isolates (29.5\%), 16 isolates (20.5\%), nine isolates (11.5\%), five isolates (6.4\%), 19 isolates (24.4\%), 23 isolates (29.5\%), and 22 isolates (28.2\%) respectively. Although the genetic diversity of group D isolates was revealed by MLST, the group D isolates harbored iutA (10 isolates, 71.4\%), iss (6 isolates, 42.9\%), cvaC (5 isolates, 35.7\%), tsh (3 isolates, 21.4\%), hlyF (9 isolates, 64.3\%), iroN (7 isolates, 50.0\%), and ompT (9 isolates, 64.3\%).
\end{abstract}

Conclusions: Our results indicated that E. coli isolates inhabiting the intestines of healthy broilers pose a potential risk of causing avian colibacillosis.

Keywords: Broiler, Escherichia coli, Multi-locus sequence typing, Phylogenetic grouping, Virulence-associated gene, Antimicrobial resistance

\section{Findings}

Escherichia coli is a commensal bacterium found in the intestinal microflora of animals. E. coli clones that carry and acquire specific virulence attributes can cause a broad spectrum of diseases. Avian pathogenic E. coli (APEC), the etiological agent of avian colibacillosis, causes extraintestinal infections - primarily respiratory infections, pericarditis, and septicemia in poultry. Avian colibacillosis was thought to be an opportunistic infection predisposed by stress [1]. Although the origin of APEC is not clearly defined, we speculated that APEC may originate from the healthy host's own or the same flocks' fecal flora.

\footnotetext{
* Correspondence: mototaka_hiki@nval.maff.go.jp

${ }^{1}$ National Veterinary Assay Laboratory, Ministry of Agriculture, Forestry and Fisheries, 1-15-1 Tokura, Kokubunji, Tokyo 185-8511, Japan

Full list of author information is available at the end of the article
}

The phylogenetic grouping of $E$. coli for the classification of extraintestinal pathogenic strains (groups B2 and D) and commensal strains (groups A and B1) in humans [2] has been applied to the characterization of $E$. coli strains from poultry. Previous studies have shown that group A and group D were predominant in APEC in Japan [3] and the United States [4]. On the other hand, Jakobsen et al. reported that only group A (approximately 40\%, nontypeable was counted as group A as was done in this study) was the dominant phylogenetic group among $E$. coli from healthy broilers $(n=138)$ [5]. Thus, the proportion of the phylogenetic groups might be different between APEC and E. coli from healthy broilers.

Some virulence genes are frequently found in APEC and assumed to be related to avain colibacillosis. iutA and iroN are iron transporter-encoding genes linking 
with growth in iron-poor environment. cvaC, iss, and omp $T$ are associated with serum and/or complement resistance linking to systemic infection. $t s h$ is associated with pathogenic process. $h l y F$ is associated with toxin [6-8]. However, the pathogenesis and the role of virulence genes in avian colibacillosis have been obscure. Therefore, prevalence of these genes was analyzed as the virulence-associated genes in $E$. coli isolates from healthy broilers in this study, to compare with that of APEC in Japan.

Molecular analyses, such as multi-locus sequence typing (MLST) of E. coli $[9,10]$, may provide additional epidemiological information on $E$. coli isolates from healthy broilers when used in combination with phylogenetic grouping or virulence profiling.

We have revealed previously that the resistance rates to ampicillin and enrofloxacin of APEC strains were higher than those of $E$. coli isolated from healthy broilers, suggesting that the antimicrobial resistance profile of APEC was different from that of $E$. coli isolated from healthy broilers. Furthermore, several studies have shown that phylogenetic groups were related to the antimicrobial resistance of $E$. coli isolates of human origin [5,11-13].

The aim of our study was to investigate possible associations of $E$. coli isolates from fecal samples of healthy broilers with APEC in Japan using phylogenetic grouping, virulence-associated gene profiling, MLST, and antimicrobial resistance profiling.

\section{Material and methods \\ Bacteria}

Ninety-six $E$. coli isolates were collected from 53 fecal samples from healthy broilers in 22 prefectures across Japan under the Japanese Veterinary Antimicrobial Resistance Monitoring System (JVARM) in 2009. Fresh fecal samples were collected from two or three healthy broilers at the different farms in each prefecture as previously described [14]. E. coli was isolated from the fecal samples using desoxycholate-hydrogen sulfate-lactose agar (Eiken Co., Ltd., Japan). Candidate colonies were identified biochemically using a commercially available kit (API20E, bioMe'rieux, Marcy-l'Etoile, France). The isolates were then stored in $10 \%$ skimmed milk (Wako Pure Chemical Industries, Ltd., Japan) at $-80^{\circ} \mathrm{C}$ until use.

\section{Genotyping}

The phylogenetic group (A, B1, B2, and D) of each isolate was determined by multiplex PCR as described by Clermont et al. [2]. Detection of the seven virulenceassociated, iutA [15], iss [8], cvaC [8], tsh [15], hlyF [6],

Table 1 Virulence profiles and antimicrobial resistance of $E$. coli from healthy broilers

\begin{tabular}{|c|c|c|c|c|c|}
\hline \multirow[b]{2}{*}{$\begin{array}{l}\text { Virulence-associated } \\
\text { gene/antimicrobial }\end{array}$} & \multicolumn{4}{|c|}{ No. (\%) of positive strains } & \multirow[b]{2}{*}{$\begin{array}{c}\text { Total } \\
(n=78)\end{array}$} \\
\hline & $\begin{array}{c}\text { A } \\
(n=35)\end{array}$ & $\begin{array}{c}\text { B1 } \\
(n=28)\end{array}$ & $\begin{array}{c}\text { B2 } \\
(n=1)\end{array}$ & $\begin{array}{c}D \\
(n=14)\end{array}$ & \\
\hline iutA & $6(17.1)^{\mathrm{a}}$ & $7(25.0)^{a}$ & & $10(71.4)^{b}$ & $23(29.5)$ \\
\hline iss & $2(5.7)^{\mathrm{a}}$ & $8(28.6)$ & & $6(42.9)^{b}$ & $16(20.5)$ \\
\hline crac & $2(5.7)^{a}$ & $2(7.1)^{\mathrm{a}}$ & & $5(35.7)^{b}$ & $9(11.5)$ \\
\hline tsh & $1(2.9)$ & $1(3.6)$ & & $3(21.4)$ & $5(6.4)$ \\
\hline iroN & $4(11.4)^{a}$ & $8(28.6)$ & & $7(50.0)^{b}$ & $19(24.4)$ \\
\hline hlyF & $4(11.4)^{a}$ & $9(32.1)$ & & $9(64.3)^{b}$ & $22(28.2)$ \\
\hline ompT & $5(14.3)^{a}$ & $9(32.1)$ & & $9(64.3)^{b}$ & $23(29.5)$ \\
\hline Ampicillin & $16(45.7)$ & $10(35.7)$ & 1 & $7(50.0)$ & $34(43.6)$ \\
\hline Cefazolin & $6(17.1)$ & $4(14.3)$ & & $4(28.6)$ & $14(17.9)$ \\
\hline Ceftiofur & $4(11.4)$ & $4(14.3)$ & & $4(28.6)$ & $12(15.4)$ \\
\hline Dihydrostreptomycin & $11(31.4)$ & $10(35.7)$ & & $7(50.0)$ & $28(35.9)$ \\
\hline Gentamicin & $2(5.7)$ & $1(3.6)$ & & & $3(3.8)$ \\
\hline Kanamycin & $7(20.0)$ & $2(7.1)$ & & $3(21.4)$ & $12(15.4)$ \\
\hline Oxytetracycline & $19(54.3)$ & $13(46.4)$ & 1 & $9(64.3)$ & $42(53.8)$ \\
\hline Chloramphenicol & $5(14.3)$ & $4(14.8)$ & & $1(7.1)$ & $10(12.8)$ \\
\hline Colistin & $1(2.9)$ & & & & $1(1.3)$ \\
\hline Nalidixic acid & $11(31.4)$ & $10(35.7)$ & & $9(64.3)$ & $30(38.5)$ \\
\hline Enrofloxacin & $5(14.3)$ & $4(14.3)$ & & $1(7.1)$ & $10(12.8)$ \\
\hline Trimethoprim & $9(25.7)$ & $12(42.9)$ & & $3(21.4)$ & $24(30.8)$ \\
\hline
\end{tabular}

A significant difference $(P<0.05)$ in prevalence was observed between ${ }^{a}$ and ${ }^{b}$. 
iroN [6], and ompT [6], was performed by PCR. Sequence type (ST) was determined using the Achtman typing scheme [16].

\section{Antimicrobial susceptibility test}

Minimum inhibitory concentrations (MICs) were determined by the agar dilution method according to the guidelines of the Clinical and Laboratory Standards Institute (CLSI) [17]. The following antimicrobial agents were tested: ampicillin, cefazolin, ceftiofur, dihydrostreptomycin, gentamicin, kanamycin, oxytetracycline, chloramphenicol, colistin, nalidixic acid, enrofloxacin, and trimethoprim. The resistance breakpoints for cefazolin, ceftiofur, dihydrostreptomycin, kanamycin, colistin, oxytetracycline, nalidixic acid, enrofloxacin, and trimethoprim were microbiologically defined as described previously [18], and those for the other antimicrobials were defined according to CLSI guidelines [17].

\section{Statistical analysis}

Differences in the prevalence of virulence-associated genes and the antimicrobial resistance rate between phylogenetic groups were analyzed using Fisher's exact test, disregarding group B2 because only a single isolate was classified into this group. A value of $\mathrm{P}<0.05$ (two-sided) was considered statistically significant.

\section{Results and discussion}

When two isolates from a fecal sample exhibited the same ST and antimicrobial resistance type, they were considered to be a single isolate. Accordingly, 78 E. coli isolates were used for further analyses.

Table 2 Sequence types of $E$. coli from healthy broilers by virulence-associated gene profiling

\begin{tabular}{|c|c|c|c|}
\hline Phylogroup & Virulence-associated gene & $\mathbf{N}$ & ST (Total no.) \\
\hline \multirow[t]{7}{*}{ A } & iutA-iss-cvaC-tsh-iroN-ompT-hlyF & 1 & $1421(1)$ \\
\hline & iutA-cvaC-iroN-ompT-hlyF & 1 & $1564(1)$ \\
\hline & iss-iroN-ompT-hlyF & 1 & $48(1)$ \\
\hline & iroN-ompT-hlyF & 1 & $1286(1)$ \\
\hline & iutA & 4 & $10(1)^{\mathrm{a}}, 48(1), 93(1)^{\mathrm{a}}, 2469(1)$ \\
\hline & ompT & 1 & $\operatorname{ND}(1)^{*}$ \\
\hline & None & 26 & $\begin{array}{l}10(11)^{a}, 752(1), 1286(1), 1630(1), 2223(2), \\
2461(1), 2462(1), 2463(2), 2465(1), 2466(2), \\
2470(1), 2471(1), 2378(1)\end{array}$ \\
\hline \multirow[t]{8}{*}{ B1 } & iutA-iss-cvaC-iroN-ompT-hlyF & 1 & $58(1)$ \\
\hline & iutA-iss-tsh-iroN-ompT-hlyF & 1 & $1079(1)$ \\
\hline & iss-cvaC-iroN-ompT-hlyF & 1 & $154(1)$ \\
\hline & iutA-iss-iroN-ompT-hlyF & 2 & $155(1)^{a}, 156(1)$ \\
\hline & iss-iroN-ompT-hlyF & 3 & $155(3)^{a}$ \\
\hline & iutA-ompT-hlyF & 1 & $101(1)^{a}$ \\
\hline & iutA & 2 & $155(1)^{a}, 2472(1)$ \\
\hline & None & 17 & $\begin{array}{l}155(4)^{\mathrm{a}}, 295(1), 453(2), 533(1), 641(1), 1079(1), \\
1724(1), 1730(1), 1771(1), 2464(1), 2473(1), \\
2475(1), 2476(1)\end{array}$ \\
\hline B2 & & 1 & $2474(1)$ \\
\hline \multirow[t]{10}{*}{$D$} & iutA-iss-cvaC-tsh-iroN-ompT-hlyF & 1 & $69(1)^{a}$ \\
\hline & iutA-iss-cvaC-iroN-ompT-hlyF & 2 & $117(2)^{a}$ \\
\hline & iss-cvaC-tsh-iroN-ompT-hlyF & 1 & $196(1)$ \\
\hline & iutA-cvaC-iroN-ompT-hlyF & 1 & $117(1)^{\mathrm{a}}$ \\
\hline & iutA-tsh-iroN-ompT-hlyF & 1 & $57(1)$ \\
\hline & iutA-iss-iroN-ompT-hlyF & 1 & $117(1)^{a}$ \\
\hline & iutA-iss-ompT-hlyF & 1 & $2309(1)$ \\
\hline & iutA-ompT-hlyF & 1 & $2477(1)$ \\
\hline & iutA & 2 & $350(1), 420(1)$ \\
\hline & None & 3 & $297(2), 457(1)$ \\
\hline
\end{tabular}

*ND: not determined because the gene for MLST could not be amplified by PCR. a: common STs of APEC isolates. 
Of the 78 isolates, 35 (44.9\%), 28 (35.9\%), one (1.3\%), and $14(17.9 \%)$ isolates belonged to groups A, B1, B2, and $\mathrm{D}$, respectively (Table 1 ). Thus, groups $\mathrm{A}$ and $\mathrm{B} 1$ accounted for approximately $80 \%$ (63/78) of the strains isolated from healthy broilers. Our previous report showed that only $53.9 \%(48 / 89)$ of APEC strains from colibacillosis-affected broilers belonged to those groups [3] and this proportion was significantly low compared with this study $(\mathrm{p}=0.0003)$. In addition, the proportion $(17.9 \%)$ of group D isolates from the healthy broilers examined in this study was lower than that (44.9\%) previously isolated from APEC-infected broilers [3]. The proportion of the phylogenetic groups in E. coli isolated from healthy broilers in this study is different from that in the APEC strains in Japan.

iutA, iss, cvaC, tsh, iroN, ompT, and $h l y F$ were found in 23 isolates $(29.5 \%), 16$ isolates $(20.5 \%)$, nine isolates (11.5\%), five isolates (6.4\%), 19 isolates (24.4\%), 23 isolates (29.5\%), and 22 isolates (28.2\%) respectively (Table 1 ). iut A and $c v a C$ were more frequently associated with group $\mathrm{D}$ isolates than groups $\mathrm{A}(\mathrm{P}=0.001$ and 0.015$)$ and $\mathrm{B} 1$ $(\mathrm{P}=0.007$ and 0.031$)$ isolates, whereas iss, hlyF, iroN and $o m p T$ were more frequently associated with group $\mathrm{D}$ isolates than group A $(\mathrm{P}=0.004,0.0004,0.007$, and 0.001 ) isolates. Group D, which is the dominant phylogenetic group in APEC strains in Japan [3], may show pathogenicity to poultry compared with the commensal groups, such as group A and B1.

All the 78 isolates were classified into 46 STs (Table 2). Among the 46 STs identified in this study, six (ST10, 69, $93,101,117$, and 155) were previously reported in APEC isolates $[9,19]$. In this study, among the 28 isolates belonging to the six STs, iutA, iss, cvaC, tsh, hlyF, iroN, and omp $T$ were detected in 10 isolates (35.7\%), eight isolates (28.6\%), four isolates $(14.3 \%)$, one isolate (3.6\%), 10 isolates (35.7\%), 9 isolates (32.1\%), and 10 isolates (35.7\%) respectively. In particular, ST117 was reported in APEC isolated in Japan [9]. All ST117 strains belonged to group $\mathrm{D}$ and possessed virulence-associated genes (Table 2). These results reinforced the hypothesis that the intestinal tract of healthy broilers might be an important natural reservoir for APEC.

A difference in the resistance rates between APEC strains of the previous study [10] and E. coli isolated from healthy broilers of this study was observed. The resistance rates against ampicillin $(\mathrm{P}=0.00002)$, kanamycin $(\mathrm{P}=0.006)$, and oxytetracycline $(\mathrm{P}=0.005)$ of $E$. coli isolated from healthy broilers were significantly lower than those of APEC [10]. Our previous study showed that the resistance rates against ampicillin and enrofloxacin were higher in APEC strains than in isolates from healthy broilers [10]. Although the higher resistance rate to enrofloxacin was not observed in this study, the $1116.7-\mathrm{kg}$ enrofloxacin was used for the treatment of broilers in
2009 (National Veterinary Assay Laboratory, 2009). In Japan, oxytetracycline has been widely used for the treatment of bacterial infection in poultry, ampicillin and kanamycin as well as enrofloxacin have been approved for the treatment of avian colibacillosis.

\section{Conclusion}

We demonstrated that the proportion of the phylogenetic groups in E. coli isolated from healthy broilers is different from that in APEC strains in Japan. On the other hand, even though group D strains accounted for a minor portion of $E$. coli isolates from healthy broilers, group D strains frequently possessed virulence-associated genes. Thus, E. coli isolates from the intestines of healthy broilers pose a potential risk of causing avian colibacillosis.

\section{Competing interests}

The authors declare that they have no competing interests.

\section{Authors' contributions}

$\mathrm{MH}$ conceived the study and the study design, interpreted the data, and drafted the manuscript. MU helped draft the manuscript. TA helped carry out the determination of phylogenetic groups and virulence-associated genes. MK, MT, SI, HS, AK, and TA helped draft the manuscript. All authors have read and approved the final manuscript.

\section{Acknowledgements}

We thank the staff of Livestock Hygiene Service Centers across Japan for providing E. coli isolates. This work was supported in part by a grant-in-aid from the Japanese Ministry of Health, Labour and Welfare.

\section{Author details}

${ }^{1}$ National Veterinary Assay Laboratory, Ministry of Agriculture, Forestry and Fisheries, 1-15-1 Tokura, Kokubunji, Tokyo 185-8511, Japan. ${ }^{2}$ Laboratory of Food Microbiology and Food Safety, Department of Health and Environmental Sciences, School of Veterinary Medicine, Rakuno Gakuen University, Ebetsu, Hokkaido, Japan. ${ }^{3}$ Food Safety and Consumer Affairs Bureau, Ministry of Agriculture, Forestry and Fisheries, 1-2-1 Kasumigaseki, Chiyoda-ku, Tokyo 100-8950, Japan. ${ }^{4}$ The United Graduate School of Veterinary Sciences, Gifu University, Gifu-shi, Gifu, Japan.

Received: 27 February 2014 Accepted: 20 June 2014 Published: 10 July 2014

\section{References}

1. Dziva F, Stevens MP: Colibacillosis in poultry: unravelling the molecular basis of virulence of avian pathogenic Escherichia coli in their natural hosts. Avian Pathol 2008, 37:355-366.

2. Clermont $\mathrm{O}$, Bonacorsi S, Bingen E: Rapid and simple determination of the Escherichia coli phylogenetic group. Appl Environ Microbiol 2000, 66:4555-4558

3. Asai T, Masani K, Sato C, Hiki M, Usui M, Baba K, Ozawa M, Harada K, Aoki H, Sawada T: Phylogenetic groups and cephalosporin resistance genes of Escherichia coli from diseased food-producing animals in Japan. Acta Vet Scand 2011, 53:52.

4. Johnson TJ, Wannemuehler $Y$, Johnson SJ, Stell AL, Doetkott $C$, Johnson JR, Kim KS, Spanjaard L, Nolan LK: Comparison of extraintestinal pathogenic Escherichia coli strains from human and avian sources reveals a mixed subset representing potential zoonotic pathogens. Appl Environ Microbiol 2008, 74:7043-7050.

5. Jakobsen L, Kurbasic A, Skjot-Rasmussen L, Ejrnaes K, Porsbo LJ, Pedersen K, Jensen LB, Emborg HD, Agerso Y, Olsen KE, Aarestrup FM, Frimodt-Moller N, Hammerum AM: Escherichia coli isolates from broiler chicken meat, broiler chickens, pork, and pigs share phylogroups and antimicrobial resistance with community-dwelling humans and patients with urinary tract infection. Foodborne Pathog Dis 2010, 7:537-547. 
6. Johnson TJ, Wannemuehler Y, Doetkott C, Johnson SJ, Rosenberger SC, Nolan LK: Identification of minimal predictors of avian pathogenic Escherichia coli virulence for use as a rapid diagnostic tool. J Clin Microbiol 2008, 46:3987-3996

7. Dho-Moulin M, Fairbrother JM: Avian pathogenic Escherichia coli (APEC). Vet Res 1999, 30:299-316.

8. Delicato ER, de Brito BG, Gaziri LC, Vidotto MC: Virulence-associated genes in Escherichia coli isolates from poultry with colibacillosis. Vet Microbiol 2003, 94:97-103.

9. Ozawa M, Baba K, Asai T: Molecular typing of avian pathogenic Escherichia coli 078 strains in Japan by using multilocus sequence typing and pulsed-field gel electrophoresis. J Vet Med Sci 2010, 72:1517-1520.

10. Ozawa M, Harada K, Kojima A, Asai T, Sameshima T: Antimicrobial susceptibilities, serogroups, and molecular characterization of avian pathogenic Escherichia coli isolates in Japan. Avian Dis 2008, 52:392-397.

11. Bukh AS, Schonheyder HC, Emmersen JM, Sogaard M, Bastholm S, Roslev P: Escherichia coli phylogenetic groups are associated with site of infection and level of antibiotic resistance in community-acquired bacteraemia: a 10 year population-based study in Denmark. J Antimicrob Chemother 2009, 64:163-168.

12. Graziani C, Luzzi I, Corro M, Tomei F, Parisi G, Giufre M, Morabito S, Caprioli A, Cerquetti M: Phylogenetic background and virulence genotype of ciprofloxacin-susceptible and ciprofloxacin-resistant Escherichia coli strains of human and avian origin. J Infect Dis 2009, 199:1209-1217.

13. Kawamura-Sato K, Yoshida R, Shibayama K, Ohta M: Virulence genes, quinolone and fluoroquinolone resistance, and phylogenetic background of uropathogenic Escherichia coli strains isolated in Japan. Jpn J Infect Dis 2010, 63:113-115.

14. Hiki M, Usui M, Kojima A, Ozawa M, Ishii Y, Asai T: Diversity of plasmid replicons encoding the bla (CMY-2) gene in broad-spectrum cephalosporin-resistant Escherichia coli from livestock animals in Japan. Foodborne Pathog Dis 2013, 10:243-249.

15. Moulin-Schouleur M, Reperant M, Laurent S, Bree A, Mignon-Grasteau S, Germon P, Rasschaert D, Schouler C: Extraintestinal pathogenic Escherichia coli strains of avian and human origin: link between phylogenetic relationships and common virulence patterns. J Clin Microbiol 2007, 45:3366-3376.

16. Wirth $T$, Falush D, Lan R, Colles F, Mensa P, Wieler LH, Karch H, Reeves PR, Maiden MC, Ochman H, Achtman M: Sex and virulence in Escherichia coli: an evolutionary perspective. Mol Microbiol 2006, 60:1136-1151.

17. CLSI: Clinical and Laboratory Standards Institute. 2013. Performance Standards for Antimicrobial Disk and Dilution Susceptibility Tests for Bacteria Isolated From Animals; Approved standard-Fourth edition and supplement. Wayne, PA: CLSI document VET01-A4 and VET01-S2; 2013.

18. Kojima A, Asai T, Ishihara K, Morioka A, Akimoto K, Sugimoto Y, Sato T, Tamura Y, Takahashi T: National monitoring for antimicrobial resistance among indicator bacteria isolated from food-producing animals in Japan. J Vet Med Sci 2009, 71:1301-1308.

19. Martinez-Medina M, Garcia-Gil J, Barnich N, Wieler LH, Ewers C: Adherent-invasive Escherichia coli phenotype displayed by intestinal pathogenic E. coli strains from cats, dogs, and swine. Appl Environ Microbiol 2011, 77:5813-5817.

doi:10.1186/2046-0481-67-14

Cite this article as: Hiki et al.: Phylogenetic grouping, epidemiological typing, analysis of virulence genes, and antimicrobial susceptibility of Escherichia coli isolated from healthy broilers in Japan. Irish Veterinary Journal 2014 67:14.

\section{Submit your next manuscript to BioMed Central and take full advantage of:}

- Convenient online submission

- Thorough peer review

- No space constraints or color figure charges

- Immediate publication on acceptance

- Inclusion in PubMed, CAS, Scopus and Google Scholar

- Research which is freely available for redistribution 